\section{Design and testing of a miniature broadband frequency domain photon migration instrument}

\author{
Keun-Sik No, ${ }^{a}$ Richard Kwong, ${ }^{a}$ Pai H. Chou, ${ }^{b}$ and \\ Albert Cerussi ${ }^{\mathrm{a}, *}$ \\ a University of California, Irvine, Beackman Laser Institute \\ Laser Medical and Microbeam Program, 1110 Health \\ Sciences Road, East, Irvine, California 92612 \\ ${ }^{\mathrm{b}}$ University of California, Irvine, Electrical Engineering and \\ Computer Science, 444F Engineering Tower, Irvine, \\ California 92697
}

\begin{abstract}
A board-level broadband frequency domain photon migration (mini-FDPM) instrument has been constructed to replace a conventional network-analyzerbased FDPM instrument. The mini-FDPM instrument with four wavelengths $(681,783,823$, and $850 \mathrm{~nm})$, matches conventional FDPM instrument in performance $(-88 \mathrm{dBm}$ noise level, $100 \mathrm{~dB}$ dynamic range) and bandwidth $(1 \mathrm{GHz})$, and recovers the same optical properties within about $6 \%$ in absorption and $4 \%$ in reduced scattering for liquid phantoms covering a wide range of relevant optical properties. Compared to the conventional FDPM instrument, the mini-FDPM instrument is more than $5 \times$ faster ( 200 ms per 401 modulation frequencies) and several orders of magnitude less in size and cost. Standard fiberoptic-based probes can be used with the mini-FDPM instrument, which increases applications in a number of clinically relevant measurement scenarios. By drastically reducing size and cost, FDPM miniaturization lowers barriers to access and will help promote FDPM in clinical research problems. The mini-FDPM instrument forms the core of a modular broadband diffuse optical spectroscopy instrument that can be used for a variety of clinical problems in imaging and functional monitoring (i.e., breast/ skin cancer, brain activation, and exercise physiology).

(c) 2008 Society of Photo-Optical Instrumentation Engineers. [DOI: $10.1117 / 1.2998473]$
\end{abstract}

Keywords: diffuse optical spectroscopy; near-infrared; photon migration; tissue spectroscopy; frequency domain photon migration (FDPM).

Paper 08059LR received Feb. 18, 2008; revised manuscript received Jul. 17, 2008; accepted for publication Aug. 26, 2008; published online Oct. 29, 2008.

Frequency domain photon migration (FDPM) is an established technique for measuring tissue optical properties (i.e., absorption, $\mu_{a}$, and reduced scattering, $\left.\mu_{s}^{\prime}\right) .{ }^{1}$ The FDPM technique uses light sources modulated at tens to hundreds of $\mathrm{MHz}$ to measure both the amplitude and the phase shift of multiply scattered light. Diffusion-based models are used to calculate absolute absorption and scattering tissue optical properties from these phase and amplitude measurements. Within the near-infrared (NIR) spectral range

*Tel: 949-824-8838; E-mail: acerussi@uci.edu.
(650 to $1000 \mathrm{~nm}$ ) where FDPM is routinely employed, the tissue concentrations of oxygenated $\left(\mathrm{ctO}_{2} \mathrm{Hb}\right)$ and deoxygenated hemoglobins $(\mathrm{ctHHb})$, water $\left(\mathrm{ctH}_{2} \mathrm{O}\right)$, and bulk lipid may be calculated from tissue absorption spectra. FDPM techniques can separate the effects of absorption from scattering using a single spatial location, which is important because NIR light is strongly multiply scattered by tissues.

Technical challenges and practical limitations have prevented the widespread use of FDPM. In contrast to the complexity of FDPM, steady-state tissue spectroscopy methods are common because off-the-shelf systems are easily assembled from commercial vendors. Substantially fewer commercial FDPM devices are available, although at high cost. FDPM techniques are further complicated by introducing broadband FDPM methods, where the light sources are modulated over a range of frequencies. Increasing modulation bandwidth improves recovered optical property accuracy in both spectroscopy and imaging. 2,3

A broadband FDPM instrument used in combination with a steady-state spectroscopy instrument to cover the entire NIR spectral range has been involved in several pilot clinical studies. ${ }^{4}$ This instrument, the laser breast scanner (LBS), represents a fairly standardized instrument platform. The core of the FDPM instrument consists of a conventional network analyzer to generate and detect modulated radio frequency (RF) currents. For each laser diode (currently six), the network analyzer generates RF frequencies in series from $50 \mathrm{MHz}$ to $1 \mathrm{GHz}$ at $15-\mathrm{dBm}$ electrical power. Laser diodes are DC-biased using a separate current source. An avalanche photo diode (APD) detects the diffuse reflectance from the tissue, sends the RF electronic signal back to the network analyzer, and compares this signal to a reference. The network analyzer measures the reflectance attenuation and phase shift between transmitter and receiver as functions of modulation frequency.

However, this network-analyzer-based FDPM instrument suffers from a number of technical limitations. First, the instrument is constructed using general-purpose commercial electronics and is therefore both expensive $(\sim \$ 60 \mathrm{k}$ total $)$ and large in size. Second, the current FDPM instrument has run up against temporal performance limits. Sweeping 401 modulation frequencies takes approximately $1 \mathrm{~s}$, but most of this time is wasted due to communication delays. Third, the high cost and large size of the instrument impedes expansion into multichannel imaging devices and increases barriers to access.

Our goal is to construct a miniature version of this LBS instrument without sacrificing any broadband information content, either spectral or temporal. The key element of this goal is a board-level FDPM instrument (mini-FDPM), as shown in Fig. 1. ${ }^{5}$ To reduce cost and size, we replaced the expensive, bulky network analyzer with a compact, customdesigned frequency-sweeping circuit. By replacing commercial off-the-shelf technologies (i.e., current sources, RF switches, etc.) with chip-based broadband communication electronics and processors, we miniaturized the FDPM instrument from a cart-based rack down to a portable unit that costs about $\$ 500$ in parts. Our broadband circuit design modulates from $10 \mathrm{MHz}$ to $1 \mathrm{GHz}$. A built-in 100 base/T Ethernet interface supports fast data transfer rates and connection with a

1083-3668/2008/13(5)/050509/3/\$25.00 @ 2008 SPIE 


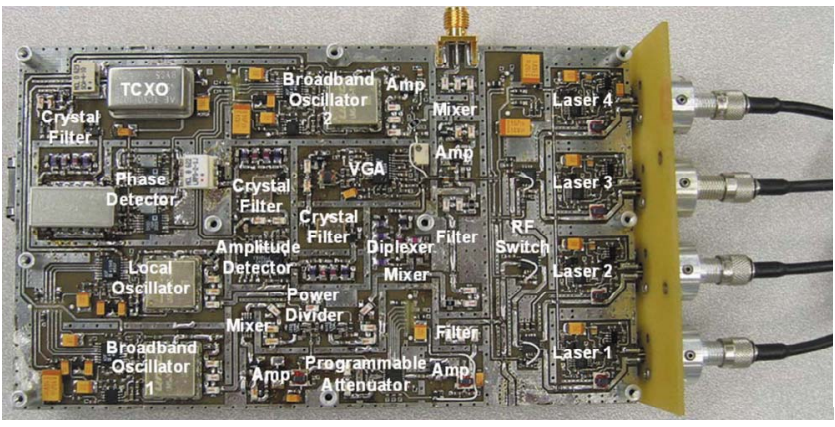

Fig. 1 Labeled photograph of the top of the circuit board. The laser diodes (right) and are connected to an optical fiber from a handheld probe.

host computer or other mini-FDPM instruments to form a network. The measurement for 401 modulation frequencies was reduced from $1 \mathrm{~s} /$ wavelength down to $0.2 \mathrm{~s} /$ wavelength; reducing the number of frequencies results in a linear decrease in measurement time.

The mini-FDPM instrument consists of a signal generator, a laser light source and driver, and an optical detector. Each component is connected with electronic filters and amplifiers to isolate signals and increase signal-to-noise ratio. The source frequency band $(10 \mathrm{MHz}$ to $1 \mathrm{GHz})$ is too broad to be generated directly from oscillators, so instead a fixed $3-\mathrm{GHz}$ frequency is mixed with a variable frequency in the 2 to $2.94 \mathrm{GHz}$ band. A phase-rocked loop (PLL) generates precise and stable frequencies by combining the flexibility of a voltage-controlled oscillator (VCO) with the stability of a $10-\mathrm{MHz}$ temperature-compensated crystal oscillator (TCXO).

The wavelengths of the four lasers were $681,783,823$, and $850 \mathrm{~nm}$, which matches the laser diodes in the networkanalyzer-based FDPM instrument. We implemented two different types of automatic power control (APC) circuits, one for the common laser diode cathode and photodiode anode on case (CCA)-type laser, and the other for the common laser diode anode and photodiode cathode on case (CAC) type. The APC circuit uses precise power monitoring feedback from the photodiode within the laser diode package. It compares the voltage induced by the monitoring current from the photodiode with the voltage induced by a digital potentiometer. By controlling the digital potentiometer value, we can carefully control laser optical power. With APC, we can modulate the laser without saturation (i.e., clipping the laser output). The average laser power was $20 \mathrm{~mW}$.

RF photocurrents from the APD are detected using a custom heterodyne circuit. The demodulated RF signal from the APD mixes with a $1.955-\mathrm{GHz}$ to $2.905-\mathrm{GHz}$ oscillator and generates $2.955 \mathrm{GHz}$ as an intermediate frequency (IF). The IF signal is then filtered and downconverted to $45 \mathrm{MHz}$ to eliminate cross-talk with the source band. This heterodyne structure reduces spurious signals with a single filter despite the broad frequency range of the photocurrents. A logarithmic amplifier and phase detector then measures the power and phase shift of the 45-MHz signal. A 16-bit multiport control unit with an integrated fast Ethernet (10/100 base/T) interface controls the system hardware and gathers the data from the amplitude and phase detector chips with a built-in analogto-digital converter (ADC).
The performance levels of the mini- and network-analyzer FDPM instruments are similar. The noise levels of networkanalyzer FDPM and mini-FDPM instruments are $-95 \mathrm{dBm}$ and $-88 \mathrm{dBm}$, respectively. However, the overall system noise level is currently detector limited by the APD $(-78 \mathrm{dBm})$. The dynamic range of the instruments are $120 \mathrm{~dB}$ (network analyzer) and $100 \mathrm{~dB}$ (mini-FDPM). Similarly, the dynamic range is again detector limited $(85 \mathrm{~dB})$. Both designs use the same handheld probe; this handheld probe included source optical fibers for the lasers and an APD. The stability of both instruments was measured using the same APD by fixing the handheld probe onto a standard tissue phantom and measuring continuously for $1 \mathrm{~h}$. The phase drift was $0.02 \mathrm{deg}$ and $0.13 \mathrm{deg}$ for the standard and mini-FDPM instruments, respectively. The mini-FDPM phase drift compares favorably with the first-generation FDPM phase drift $(0.3 \mathrm{deg}$ over $30 \mathrm{~min})$. The amplitude drift was $2 \%$ and $6 \%$ for the standard and mini-FDPM instruments, respectively. This is to be compared with a $3 \%$ drift over 30 min for the first-generation FDPM instrument. The drift errors translated into optical properties of $1.3 \%$ and $3 \%$ for the standard and mini-FDPM instruments, respectively. The lower drift of the standard instrument is likely due to the individual temperature control circuits for each laser diode. Implementing a similar strategy for the mini-FDPM instrument should significantly reduce the drift.

We designed phantom experiments to characterize the performance in the recovery of optical properties of the miniFDPM instrument relative to the standard FDPM instrument. We fabricated 10 homogeneous liquid phantoms. Liquid phantoms (1000 mL volume each) were made with varying concentrations of Lyposin (Abbott Laboratories, Chicago, Illinois) as the scattering agent and a water-soluble dye, Nigrosin (Sigma, St. Louis, Missouri), as the absorbing agent. The phantoms were designed to emulate the optical properties of tissues typically encountered by the LBS: normal breast (50 ml Lyposin, $3 \mathrm{ml}$ Nigrosin), normal brain (60 ml Lyposin, $12 \mathrm{ml}$ Nigrosin), muscle and breast tumors (30 $\mathrm{ml} \mathrm{Ly-}$ posin, $14 \mathrm{ml} \mathrm{Nigrosin}$ ), and bone (70 $\mathrm{ml}$ Lyposin, $4 \mathrm{ml}$ Nigrosin).

We used the same handheld optical probe, for both systems. In this way, the performance of the FDPM hardware could be evaluated independently of the choice of detectors, source fibers, and probe coupling. All FDPM measurements were taken with a single source-detector distance in a reflectance semi-infinite geometry. The optical fibers were positioned on the liquid phantom surface. Instrumental phase and amplitude artifacts were calibrated using a separate liquid phantom, which was also constructed from Intralipid and Nigrosin, with known optical properties.

Figure 2 shows the recovered $\mu_{a}$ and $\mu_{s}^{\prime}$ values for both FDPM instruments. We used a Bland and Altman plot to compare the phantom optical properties measured by both instruments. ${ }^{6}$ The Bland and Altman plot compares the mean of the two measurements versus the difference of the two measurements and is generally preferred to correlation plots. The results revealed that the mini-FDPM instrument is a good substitute for the standard FDPM instrument. For all wavelengths, the average deviation between $\mu_{a}$ values was $0.0006 \mathrm{~mm}^{-1}$, and $0.03 \mathrm{~mm}^{-1}$ for $\mu_{s}^{\prime}$. The average percent differences of recovered $\mu_{a}$ and $\mu_{s}^{\prime}$ from all phantoms 


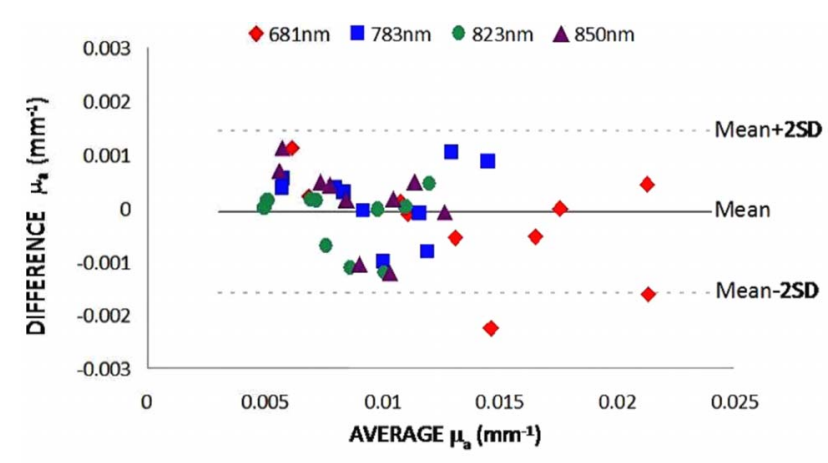

(a)

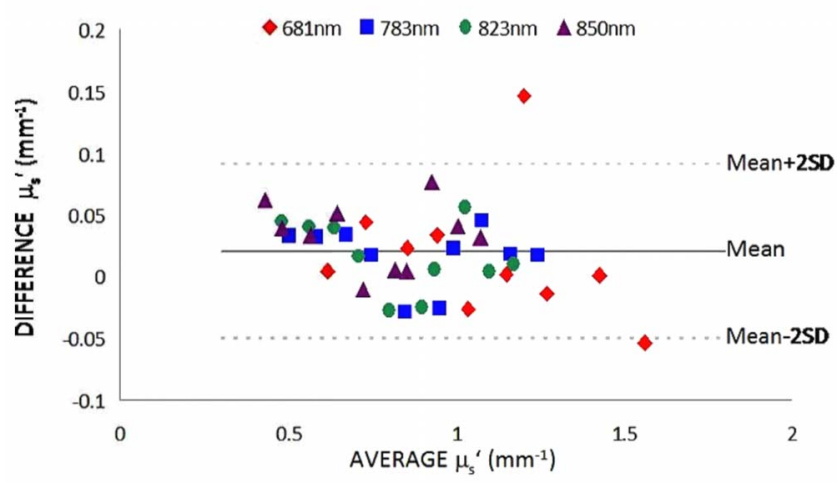

(b)

Fig. 2 Recovered optical properties of 10 liquid phantoms using both standard and mini-FDPM instruments. The comparisons are plotted using Bland and Altman plots for the recovered $\mu_{a}$ (panel a) and $\mu_{s}^{\prime}$ (panel b). In general, the mini-FDPM instrument reliably recovered the same $\mu_{a}$ and $\mu_{s}^{\prime}$ values as the standard FDPM instrument over a wide range of physiologically relevant optical properties.

between instruments were $5.9 \pm 6.3 \%$ and $3.2 \pm 3.4 \%$ at $681 \mathrm{~nm}, 5.7 \pm 3.3 \%$ and $3.4 \pm 1.7 \%$ at $783 \mathrm{~nm}, 4.7 \pm 5.4 \%$ and $3.7 \pm 2.9 \%$ at $823 \mathrm{~nm}, 7.4 \pm 5.9 \%$ and $5.2 \pm 4.1 \%$ at $850 \mathrm{~nm}$, respectively. In absolute terms, the average difference in $\mu_{a}$ was $0.0005 \mathrm{~mm}^{-1}$ for $\mu_{a}<0.01 \mathrm{~mm}^{-1}$, $0.0007 \mathrm{~mm}^{-1}$ for $0.01 \leqslant \mu_{a}<0.015 \mathrm{~mm}^{-1}$, and $0.0006 \mathrm{~mm}^{-1}$ for $\mu_{a} \geqslant 0.015 \mathrm{~mm}^{-1}$. Equivalently, the average difference in $\mu_{s}^{\prime}$ was $0.03 \mathrm{~mm}^{-1}$ for all ranges of $\mu_{s}^{\prime}$. These average difference values demonstrate that the miniFDPM instrument replicates the standard FDPM measurements across a physiologically relevant $\mu_{a}$ and $\mu_{s}^{\prime}$ values.

The mini-FDPM instrument presented here offers unique opportunities. It currently offers broadband modulation frequency capabilities that are not commercially available. The measurement speed, which is less than $200 \mathrm{~ms}$ per wavelength, can be linearly reduced by decreasing the number of modulation frequencies (currently 401). The mini-FDPM instrument may also be combined with a spectrometer system in order to perform spectrally broadband measurements." In this way, the mini-FDPM instrument forms the core of broadband diffuse optical spectroscopy and imaging (DOSI) instruments.

The mini-FDPM instrument prototype represents a modular and versatile solution to making broadband FDPM instruments readily available to the research community. The versatility and modularity is first demonstrated by the simple interfacing of the mini-FDPM instrument with any of our handheld probes developed for the standard FDPM instrument. We demonstrated within this letter that the mini-FDPM instrument interfaces with the same handheld probe we developed for our clinical breast measurements. Because the miniFDPM instrument uses standard optical fibers, virtually any multimode fiber-optic probe can interface with the miniFDPM instrument.

The mini-FDPM instrument, offers an expandable solution to increase the number of detection channels and collect spatial information in parallel. The network analyzer is a platform illsuited for practical multichannel clinical diffuse optical imaging. While the parallel collection concept is not novel, parallel detection while retaining broadband information content has not been achieved. By combining the miniFDPM instrument with a broadband spectrometer system, we envision a spectrally and temporally broadband diffuse optical spectroscopic imaging device that may be configured into many imaging geometries (i.e., handheld probe and tomographic ring).

In all essential characteristics (signal-to-noise, drift, dynamic range, and noise floor), the mini-FDPM instrument effectively replaces our conventional network-analyzer-based FDPM instrument. In addition, the recovery of optical properties is similar between instruments within about $0.0006 \mathrm{~mm}^{-1}$ for $\mu_{a}$ (over the range $0.005 \mathrm{~mm}^{-1}$ to $0.022 \mathrm{~mm}^{-1}$ ) and $0.03 \mathrm{~mm}^{-1}$ for $\mu_{s}^{\prime}$ (over the range $0.46 \mathrm{~mm}^{-1}$ to $1.5 \mathrm{~mm}^{-1}$ ). Optical property recovery was accomplished $5 \times$ faster, $120 \times$ cheaper, and with equipment over $100 \times$ smaller than with our conventional instrument.

\section{Acknowledgments}

This work was supported by the National Institutes of Health under Grant Nos. P41-RR01192 (Laser Microbeam and Medical Program-LAMMP) and U54-CA105480 (Network for Translational Research in Optical Imaging-NTROI), the National Science Foundation (CNS-0448668), and the Henry T. Nicholas Fellowship program. The authors wish to thank Bruce Tromberg for his many insightful discussions and Amanda F. Durkin for her technical assistance.

\section{References}

1. T. H. Pham, O. Coquoz, J. B. Fishkin, E. Anderson, and B. J. Tromberg, "Broad bandwidth frequency domain instrument for quantitative tissue optical spectroscopy," Rev. Sci. Instrum. 71(6), 2500-2513 (2000).

2. J. B. Fishkin, S. Fantini, M. J. VandeVen, and E. Gratton, "Gigahertz photon density waves in a turbid medium: theory and experiments," Phys. Rev. E 53(3), 2307-2319 (1996).

3. G. Gulsen, B. Xiong, O. Birgul, and O. Nalcioglu, "Design and implementation of a multifrequency near-infrared diffuse optical tomography system," J. Biomed. Opt. 11(1), 014020 (2006).

4. A. Cerussi, N. Shah, D. Hsiang, A. Durkin, J. Butler, and B. J. Tromberg, "In vivo absorption, scattering, and physiologic properties of 58 malignant breast tumors determined by broadband diffuse optical spectroscopy," J. Biomed. Opt. 11(4), 044005 (2006).

5. K. S. No and P. H. Chou, "Mini-FDPM and heterodyne mini-FDPM: handheld noninvasive breast cancer detectors based on frequencydomain photon migration," IEEE Trans. Circuits Syst., I: Regul. Pap. 52(12), 2672-2685 (2005).

6. J. M. Bland and D. G. Altman, "Statistical methods for assessing agreement between two methods of clinical measurement," Lancet 1(8476), 307-310 (1986). 